\title{
Determination of extensibility for certain types of pretzels dough
}

\author{
Mariana Gabriela Munteanu, ${ }^{*}$, Gheorghe Voicu${ }^{1}$, Elena Mădălina Ștefan ${ }^{1}$ and Gabriel \\ Alexandru Constantin ${ }^{1}$ \\ ${ }^{1}$ Politehnica University of Bucharest, Faculty of Biotechnical Systems Engineering, Romania
}

\begin{abstract}
Determination of pretzels dough is essential to analyse the technological process, the dough handling behaviour and properties of the finished product. In various processing steps such as kneading, dividing, shaping and baking the dough undergoes different mechanical deformation, including the extent, compression or shear. In the literature there are several methods for analysing these properties. These include empirical rheological methods, such as recording dough evolution during kneading, extensibility measurement or fundamental rheological methods. The study in this paper propose to investigate the behaviour of different types of test dough extensibility and make estimates of the values of the moment (time) and maximum force that wick dough is deformed to appearance of fracture. The maximum elongation of the dough wick is determined for the same values of its initial geometry as well as the other test parameters for two types of dough, one with a composition of $45 \%$ water, $10 \%$ oil and $45 \%$ flour, and another with a composition of $55 \%$ water, $10 \%$ oil and $35 \%$ flour. Preliminary results have found that a dough with more water has a greater tensile strength than the dough with the same amount of oil and less water.
\end{abstract}

\section{Introduction}

The dough extensibility is a rheological property which begins to form even during the dough kneading. If during kneading the elastic properties are not formed, then the dough will not have the proper rheological behaviour in fermentation, modelling or baking processes, [1]. In more general terms, the extensibility of the dough is its property of stretching, without breaking, [2,3]. This property does not depend only on the quality and amount of gluten in wheat flour, depends also on the amount of water added in the mixture, $[4,5]$.

The main method for determining the dough extensibility is standardized with AACC Method no. 54-10.01/ ISO 5530-2/ ICC-Standard no. 114/1 and involves stretching a dough wick and drawing extensographic curves with the help of Brabender Extender, [6].

Results on dough stretching help determine its rheological properties, the amounts of ingredients to obtain it, including the possibility of processing and baking, [7]. A very extensible dough is used in the bread making process because it is considered easier to

\footnotetext{
* Corresponding author: munteanumaya@yahooo.com
} 
process, and the less extensible dough is used for other bakery products such as pretzels, sticks, grissins, [6].

The tensile strength of the dough is recorded in the Brabender Extender system data recorder. The recording system has the role of plotting extensographic curves which allow the determination of certain characteristics:

- resistance to stretching due to maximum resistance, $\mathrm{Rm}$ and resistance to constant deformation corresponding to the time of stretching at $50 \mathrm{~mm}$, R50. Each of the average values is expressed by approximately $5 \mathrm{UE}$;

- the extensibility is due to the displacement of the dough from the time of the curve recording until the moment the sample breaks and is indicated on the extensogram by a smooth fall to zero or by a sudden fall; each of the mean values is expressed by approximately $1 \mathrm{~mm}$;

- energy is defined as the area under the curve recorded and describes the effort required to stretch the dough wick; where: $m_{a}$ represents the dough mass [mm].

$$
W=\frac{\text { Aria }}{m_{a}} 10^{3}
$$

- the $\mathrm{R} / \mathrm{E}$ ratio is noted with $\delta$ and is the ratio of maximum resistance $\mathrm{Rm}$ or resistance to constant deformation, R50 and extensibility, [8]

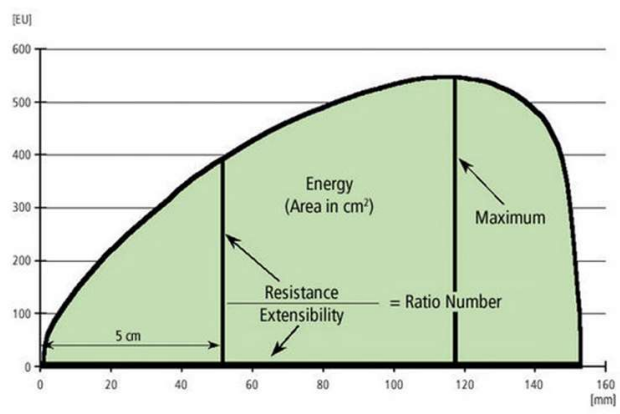

Fig. 1 Extensograph evaluation $[9,10]$

\section{Methodology}

To quantify the extensibility of the dough, the Brabender extensograph is generally used or other comparable test devices, $[6,9,11]$. In all situations, the force used to stretch the dough wicks up to breakage, when it is reached at the maximum dough extensibility level, $[12,13]$. The dough used to determine extensibility with the Brabender extensograph is obtained with the help of the Brabender farinograph, by the method AACC 54-21 (2000) and must have the characteristics of a normal consistency dough $[5,14]$. The dough is prepared from $300 \mathrm{~g}$ flour, water and 1,2\% salt, and a $150 \mathrm{~g}$ sample is divided from the total dough which is shaped like a wick (long form). The wick of dough is placed on racks dough and let rest in thermostat for 45, 90, 135 and 180 minutes. After the thermostat period, the dough wick is subject to stretching until it breaks, $[6,8]$.

Determinations result in an extension curve whose characteristics indicate the rheological properties of the dough (figure 1). The rheological properties of the dough depend on the next steps in the established technological flow, [8].

Two types of dough were prepared for the proposed study: 
- type I - dough with composition of $45 \%$ water, $10 \%$ oil from the total amount of flour;

- type II - dough with composition of $55 \%$ water and $10 \%$ oil from the total amount of flour.

Besides the amount of water and oil, to obtain each type of dough was also added the flour purchased from the trade $(1 \mathrm{~kg})$, kitchen salt $(0.012 \mathrm{~kg})$, yeast $(0.005 \mathrm{~kg})$, sugar $(0.010 \mathrm{~kg})$. Flour had a moisture of $9,56 \%$ and a hydration capacity of $65,7 \%$ which was determined with the help of the Brabender farinograph. For blending the ingredients and kneading the dough was used spiral arm and planetary motion kneader, DitoSama. Mixing the ingredients was done for 2 minutes in the first velocity stage of the kneading arm, and subsequently switched over for 10 minutes in the second stage of the velocity to carry out the kneading process of the dough. After completing the kneading, the dough was divided into pieces whose mass was $100 \mathrm{~g}$.

To perform the extensibility test, pieces of dough were moulded in the form of wicks with a length of $400 \mathrm{~mm}$ and a diameter of about $16 \mathrm{~mm}$ and placed on the fixed crosspiece of the HOUNSFIELD H1KS Mechanical Testing Machine. The apparatus used during the experiments was equipped with a hook, a force cell of $10 \mathrm{~N}$ and a clamping system for the end of wicks on the fixed crosspiece of the device. To record the maximum force that has been applied for stretching to break the dough wick was used a computer on which the Qmat software was installed.

After attaching wick on the fixed crosspiece and hanging the hook on the movable traverse, the wick was lifted vertically at a speed of $50 \mathrm{~mm} / \mathrm{min}$. The movement of the hook was made until the dough wick broke into one of the tear areas a, b, c, d, e from figure 2. At the time of breaking the dough wick in the Qmat software acquisition system the maximum force at which the breakage occurred and the hook movement vertically was recorded for each wick sample (figure 3).

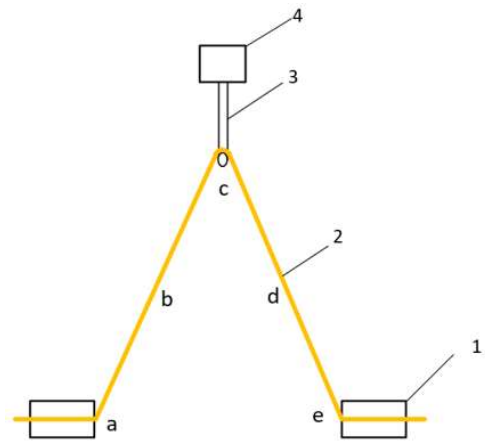

a)

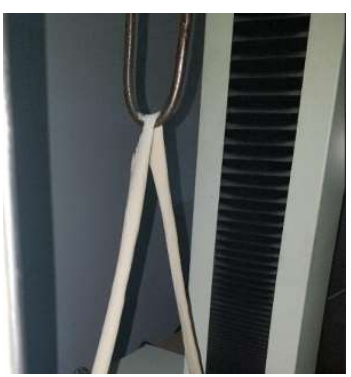

b)

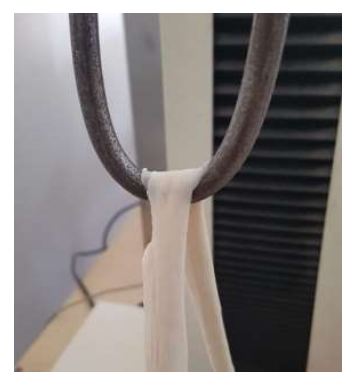

Fig. 2. a) Traction test who showing dough extensibility and breakage zones (a, b, c, d, e); 1 attaching the wick holder; 2. wick; 3. hook; 4. the force cell $(10 \mathrm{~N}) ;$. b) an example

After recording data for all samples of the two types of dough, the information has been input into Excel and processed so as to identify the moment of dough breakage and displacement from the start of the lifting stroke to the maximum breaking force. 


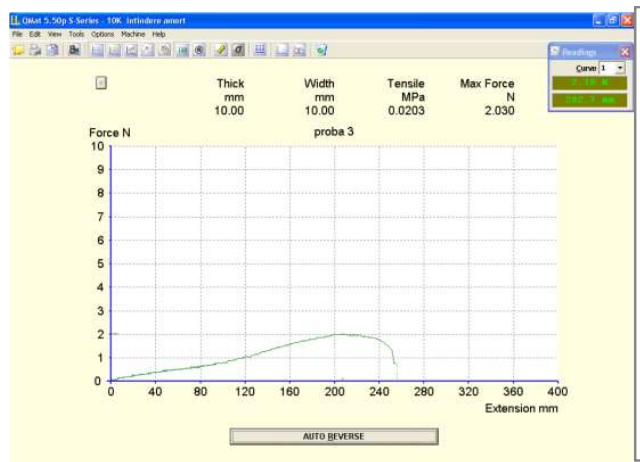

a)

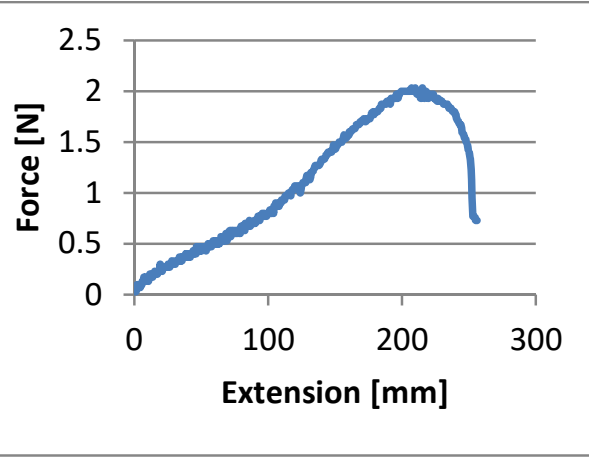

b)

Fig. 3 Example of image extracted during experiments a) Image of the Qmat software; b) Image from the Excel program

To determine the modulus of elasticity (E) the linear regression analysis of the experimental points recorded since the "zero" moment to the point where the stress-strain curve shows a sharp curve before the maximum point. This is clearly visible on the graph of experimental points traced in Excel, because it shows a roughly linear variation up to the maximum force point.

The angle of the horizontal regression line represents the modulus of elasticity (see relationship 2).

$$
E=\frac{L \cdot \operatorname{tg} \alpha}{A_{0}} \quad[\mathrm{~N} / \mathrm{m} 2]
$$

where: L- length of the wick after stretching; $\mathrm{A}_{0}$ - the initial area of the wick section.

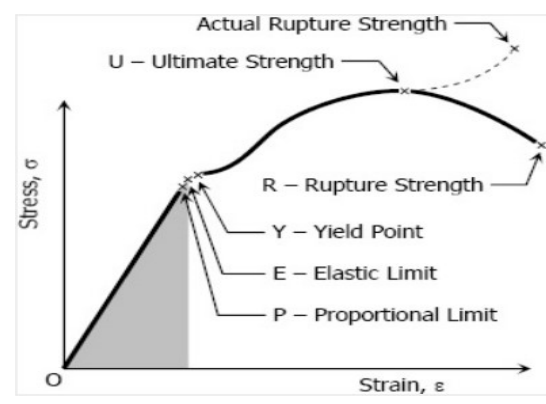

Fig. 4 Diagram "stress-strain" [15]

To determine the length of the dough corresponding to the moment of reaching the maximum force the value of the angle $\beta$ formed by it at the point "c" in Figure 1 was first found, applying the calculation scheme in Figure 5 and it was taken into account to drop $100 \mathrm{~mm}$ of the length of the wick, since the ends of the wicks have been inserted into the holder of the dough wicks. 


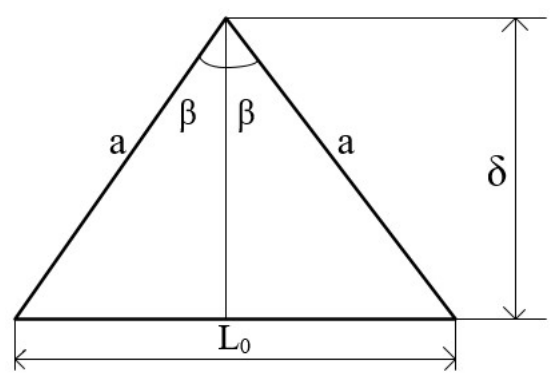

Fig. 5 Calculation scheme for determining the relative length of the dough

$$
\operatorname{tg} \beta=\frac{L_{0}}{2 \delta}
$$

where: $\mathrm{L}_{0}$ represents the length of the wick after the catch $[\mathrm{mm}] ; \delta$ represents the movement of the wick vertically $[\mathrm{mm}]$.

After finding the angle $\beta$ the value of the wick extension was calculated (L), with relationship (4) and (5).

$$
a=\frac{L_{0}}{2 s i} \quad[\mathrm{~mm}]
$$

namely,

$$
L=2 a \quad[\mathrm{~mm}]
$$

Knowing the initial length $\left(\mathrm{L}_{0}\right)$ and length at stretching $(\mathrm{L})$ of the wick, could be calculated deformation $(\varepsilon)$, and dough resistance $(\sigma)$ to stretch using relationships $(6)$ and $(7)$.

$$
\begin{gathered}
\varepsilon=\frac{2 L-L_{0}}{L_{0}} \quad[\mathrm{~mm}] \\
\sigma=\frac{F}{A}=\frac{F \cdot L}{A_{0} \cdot L_{0}} \quad\left[\mathrm{~N} / \mathrm{m}^{2}\right]
\end{gathered}
$$

\section{Results and discussions}

Experimental results recorded for maximum strength, respectively the displacement of the hook corresponding to the maximum force is shown in Table 1 and in the graphs of Figures 6 and 7. Graphs have been processed using Excel.

Table 1 The experimental results recorded following determinations

\begin{tabular}{|c|c|c|c|c|c|c|c|c|}
\cline { 2 - 9 } \multicolumn{1}{c|}{} & \multicolumn{4}{c|}{ Dough type I } & \multicolumn{4}{c|}{ Dough type II } \\
\cline { 2 - 9 } \multicolumn{1}{c|}{} & \multicolumn{4}{c|}{ Sample } & \multicolumn{4}{c|}{ Sample } \\
\cline { 2 - 9 } & $\mathbf{1}$ & $\mathbf{2}$ & $\mathbf{3}$ & $\mathbf{4}$ & $\mathbf{1}$ & $\mathbf{2}$ & $\mathbf{3}$ & $\mathbf{4}$ \\
\hline $\begin{array}{c}\text { Extension } \\
{[\mathrm{mm}]}\end{array}$ & 200 & 205 & 215.2 & 234 & 272.8 & 190 & 217 & 188.9 \\
\hline $\begin{array}{c}\text { Maximum } \\
\text { force [N] }\end{array}$ & 1.53 & 1.9 & 2.03 & 2.17 & 2.67 & 2.67 & 2.67 & 2.67 \\
\hline
\end{tabular}




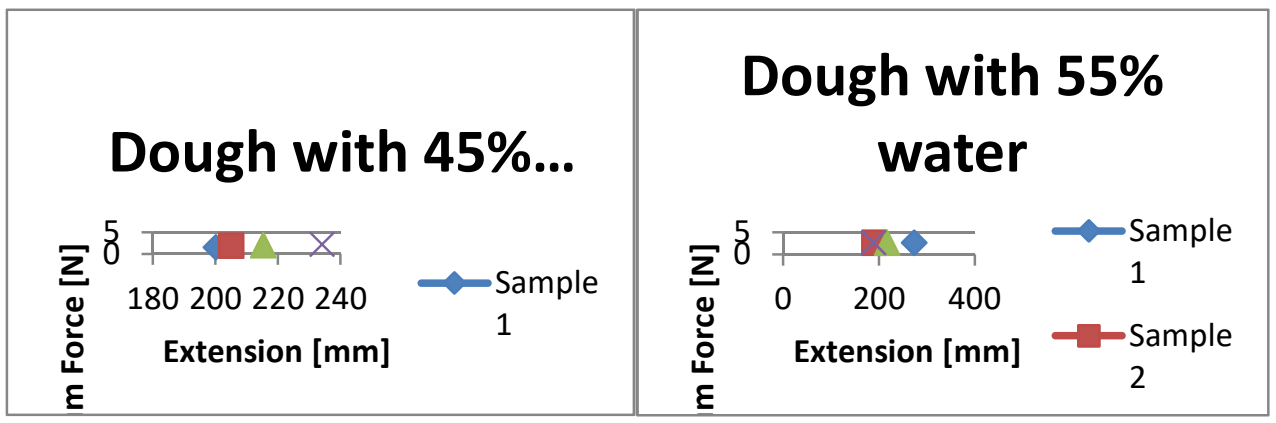

Fig. 6 Variation of force and maximum hook displacement for dough type I
Fig. 7 Variation of force and maximum hook displacement for dough type II

The charts in Figures 6 and 7 shows the variation of the force values and the maximum hook displacement for dough types I and II and from what can be seen from these graphs for type I dough, the maximum force values for the four samples fall within the range 1.53 $2.17 \mathrm{~N}$, and for type II dough, the strength values remained unchanged for all four dough samples $(2.67 \mathrm{~N})$. Even if the force values remained unchanged for type II dough, it can be seen that samples of this type of dough required a higher breaking strength compared to dough type I. In the case of hook displacement values for type I dough, they fall within a much narrower range, i.e. $200-234 \mathrm{~mm}$, compared to the hook displacement for type II dough samples, i.e. 188.9 - $272.8 \mathrm{~mm}$. By this analysis it can be pointed out that the samples of type II dough had a much greater extensibility until the moment of flowing and reaching the breaking point compared to the Type I dough samples.

Elastic modulus values (E) determined by linear regression, the length of the wick after stretching $(\mathrm{L})$, the deformation values $(\varepsilon)$ and the resistance of the dough to the stretch $(\sigma)$ are shown in Table 2.

Table 2 Physical characteristics and experimental data obtained for pretzels dough wicks

\begin{tabular}{|c|c|c|c|c|c|c|}
\hline $\begin{array}{c}\text { Dough } \\
\text { type }\end{array}$ & Sample & $\begin{array}{c}\mathbf{L 0 \cdot 1 0 - 3} \\
{[\mathbf{m}]}\end{array}$ & $\begin{array}{c}\mathbf{L} \cdot \mathbf{1 0 - 3} \\
{[\mathbf{m}]}\end{array}$ & $\boldsymbol{\varepsilon}[\mathbf{m m}]$ & $\begin{array}{c}\mathbf{E} \\
{[\mathbf{M P a}]}\end{array}$ & $\begin{array}{c}\boldsymbol{\sigma} \\
{[\mathbf{M P a}]}\end{array}$ \\
\hline \multirow{2}{*}{$\begin{array}{c}\text { Dough } \\
\text { with }\end{array}$} & 1 & 300 & 500 & 0.66 & 0.069 & 0.05 \\
\cline { 2 - 7 } & 2 & 300 & 508.5 & 0.69 & 0.91 & 0.064 \\
\cline { 2 - 7 } $\begin{array}{c}45 \% \\
\text { water }\end{array}$ & 3 & 300 & 526.3 & 0.75 & 0.094 & 0.07 \\
\cline { 2 - 7 } Dough & 4 & 300 & 566 & 0.88 & 0.1 & 0.081 \\
\cline { 2 - 7 } with & 2 & 300 & 638.28 & 1.27 & 0.072 & 0.11 \\
\cline { 2 - 7 } $55 \%$ \\
water & 3 & 300 & 500 & 0.66 & 0.123 & 0.088 \\
\cline { 2 - 7 } & 4 & 300 & 482.33 & 0.6 & 0.098 & 0.085 \\
\hline
\end{tabular}

To highlight the increase in dough extensibility along with increasing the amount of water in the dough the mean of the modulus of the elastic modulus was made and the length of the dough to the stretch and were shown in Figures 8. 


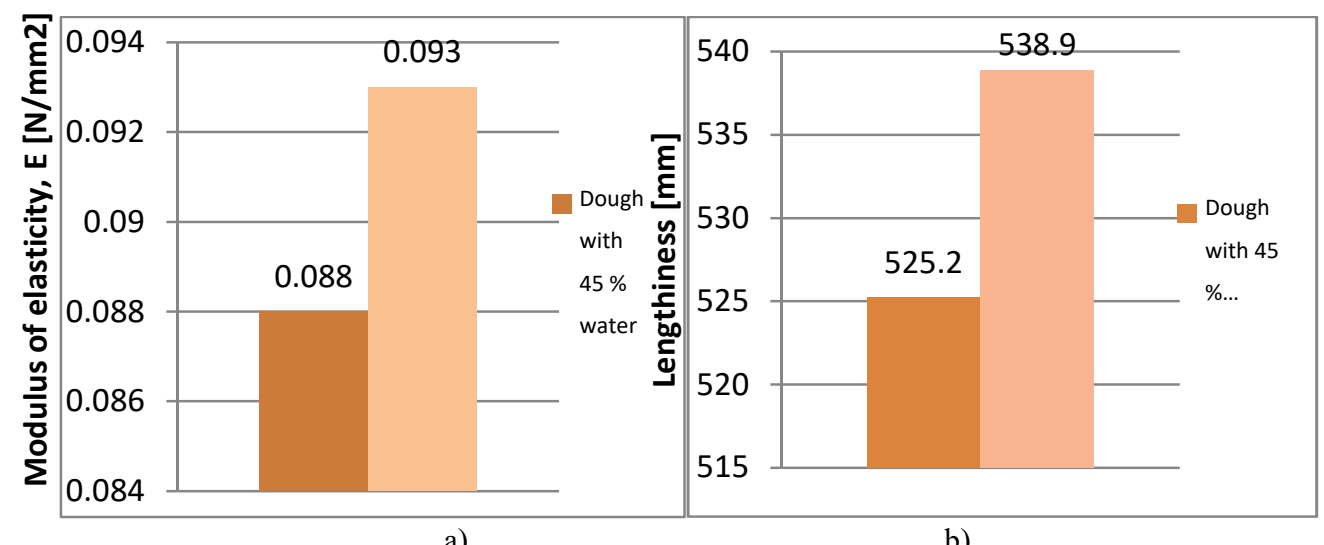

Fig. 8 a) Evidence of the average elastic modulus difference for the two types of dough; b) Evidence of the difference in the average length of the wick dough to the stretch for the two types of dough

\section{Conclusions}

The experimental data show a deformation of the dough within the limits of $0.66-0.88$ until it reaches the breaking strength for dough with $45 \%$ water and within $0.6-1.7$ for a dough with $55 \%$ water.

Modulus of elasticity of dough with $45 \%$ water and $10 \%$ oil has much lower values compared to $55 \%$ water and $10 \%$ oil.

It can be concluded that a greater amount of water in the dough leads to an increase in dough extensibility, respectively, to easier handling of the dough wicks without breaking or thinning during moulding.

A dough with less water will break faster than one with a higher water content.

\section{References}

1.E. Chiang, https://bakerpedia.com/test-dough-extensibility-why-you-need-to/, (2017)

2. ***https://www.foodtechcorp.com/tms-extensibility-fixture, accessed in April 2019

3. Gh. Constantin, Resarch on Rheological Behavior of Wheat Flour Dough for Bakery Applications - Phd. Thesis, Bucharest, (2011)

4. Wheat Marketing Center, Wheat and Flour Testing Methods: A Guide to Understanding Wheat and Flour Quality, Kansas State University, (2008)

5. M.J. Patel, S. Chakrabarti-Bell, J Food Eng, 115, 371- 383, (2013)

6. S. Chakrabarti-Bell, M.J. Patel, Int J Food Eng, 12, (2016)

7.S. Chakrabarti-Bell, M.J. Patel a, J. Ng, W.E. Hawkins, J Food Eng, 209, 36-44, (2017)

8. G.G. Codina, The rheological properties of the wheat flour dough, (Ed. AGIR, Series "Food Engineering", Bucharest, (2010)

9. ***https://www.brabender.com/en/food/products/rheometers/inspect-rheologicaldough-properties-extensograph-e/, accessed in April 2019

10.***http://www.wafermatic.com/wheattesting.html, accessed in April 2019 
11. B. Dunnewind, E.L. Sliwinski, K. Grolle, T. Van Vliet, J Texture Stud, 34, 537-560, (2004)

12. Ș. Tietze, M. Jekle, T. Becker, Trends Food Sci Technol, 57, 1-10, (2016)

13. D. Nash, S. P. Lanning. P. Fox, J. M. Martin, N. K. Blake, E. Souza, R. A Graybosch, Cereal Chem., 83, 255 - 258 (2006)

14. S. Md. Zaidul Islam, Y. Hiroaki, K. Sun-Ju, M. Chie, T. Shigenobu, H. Naoto, N. Takahiro, Food Sci. Technol. Res., 14 (2), 211 - 216 (2008)

15. ***http://www.forohistarmar.com.ar/foro/showthread.php?tid=670, accessed in April 2019 